Revista de Filosofía

ISSN: 0034-8244

http://dx.doi.org/10.5209/RESF.64271

\title{
La psyché judeoalemana ante el derecho y el Estado: Walter Benjamin y Franz Rosenzweig
}

\author{
Roberto Navarrete Alonso ${ }^{1}$
}

Recibido: 30 de enero de 2018 / Aceptado: 03 de mayo de 2018

Resumen. El artículo analiza el vínculo entre derecho y violencia en Walter Benjamin y Franz Rosenzweig. Primero, se estudia la relación entre Benjamin, Rosenzweig y Carl Schmitt, y, a continuación, la proximidad entre las concepciones benjaminiana y rosenzweiguiana de la historia y de la temporalidad mesiánica, así como la relación entre justicia, derecho y eternidad. Finalmente, se presenta la propuesta derridiana de una deconstrucción de la crítica benjaminiana de la violencia y su antecedente en La estrella de la redención.

Palabras clave: Walter Benjamin; Franz Rosenzweig; derecho; violencia; Estado.

\section{[en] The German-Jewish psyche in Law and the State: Walter Benjamin and Franz Rosenzweig}

\begin{abstract}
This paper deals with the relationship between law and violence according to Walter Benjamin and Franz Rosenzweig. Firstly, I explore the relationship between Benjamin, Rosenzweig and Carl Schmitt. Secondly, I analyze the similarities between Benjamin's and Rosenzweig's conceptions of history and messianic temporality, as well as the relationship between justice, law and eternity. Finally, I present Jacques Derrida's deconstruction of Benjamin's critique of violence and its precedent in The Star of Redemption.
\end{abstract}

Keywords: Walter Benjamin; Franz Rosenzweig; law; violence; State.

Sumario: 1. Introducción; 2. Encuentros y desencuentros; 2.1. Walter Benjamin y Carl Schmitt; 2.2. Walter Benjamin y Franz Rosenzweig; 2.3. Franz Rosenzweig y Carl Schmitt; 3. Historia, mesianismo, derecho y justicia; 3.1. Historia como catástrofe versus temporalidad mesiánica; 3.2. Justicia, derecho y eternidad; 4. Crítica de la crítica benjaminiana de la violencia: Derrida y Rosenzweig; 5. Conclusión; 6. Referencias bibliográficas.

Cómo citar: Navarrete Alonso, R. (2019): "La psyché judeoalemana ante el Derecho y el Estado: Walter Benjamin y Franz Rosenzweig”, en Revista de Filosofia 44 (1), 61-77.

Universidad Complutense de Madrid

roberto.navarrete@ucm.es 


\section{Introducción}

El presente trabajo tiene su origen en unas palabras leídas por Jacques Derrida, el 26 de abril de 1990, en un coloquio sobre "El nazismo y la "solución final"'. El pensador judío, argelino y francés, proponía en dicho contexto una lectura del ensayo de Walter Benjamin Hacia la crítica de la violencia ${ }^{3}$, de 1921. En la introducción a dicha lectura, publicada cuatro años más tarde bajo el título "Nombre de pila de Walter Benjamin" como segunda parte de su obra Fuerza de ley: el "fundamento místico de la autoridad"4, Derrida contextualizaba su interés en el célebre texto de Benjamin, en primer lugar, en el marco de un seminario sobre "Nacionalidades y nacionalismos filosóficos" y, en particular, en una serie de sesiones en las que, bajo el rótulo Kant, el judio, el alemán ${ }^{5}$, el fundador de la deconstrucción se interesó, como él mismo lo expresa, por la "psyché judeoalemana". Derrida la define como "la lógica de ciertos fenómenos de turbadora espectacularidad, reflejada ésta a su vez en ciertas grandes figuras de pensadores y escritores judíos alemanes de este siglo [es decir, para nosotros, del pasado siglo]"’. Entre estas grandes figuras menciona, por supuesto, a Walter Benjamin, pero también a Franz Rosenzweig, a quien de hecho se había referido en su seminario sobre el germano-judaísmo de Kant ${ }^{8}$. La cuestión, prosigue Derrida, tendría que ver con la existencia de "ciertas analogías, a veces de las más equívocas e inquietantes, entre los discursos de ciertos 'grandes' pensadores alemanes y ciertos 'grandes' pensadores judíos alemanes" " Entre los primeros Derrida -no olvidemos el contexto en el que está pronunciando estas palabras: un encuentro sobre el III Reich y la Endlösung-menciona a Heidegger y a Carl Schmitt, cuyo compromiso con el Estado hitleriano, sin duda, no conviene olvidar nunca -aunque tampoco, dicho sea de paso, quepa reducirlos, de manera respectiva, a la condición de filósofo y jurista de la Alemania nacionalsocialista-. Lo que Derrida afirma en concreto es que a su juicio deben explorarse con seriedad "ciertas afinidades, limitadas pero determinables, entre este texto de Benjamin [a saber, Hacia la crítica de la violencia] y ciertos textos de Carl Schmitt, o de Heidegger" ". Lo que aquí propongo es, en definitiva, dejar de lado al pensador de Meßkirch e introducir, en cambio, al de La estrella de la redención. Centraré la atención en la confrontación de las respectivas posiciones de Benjamin y Rosenzweig ante el derecho y ante el

2 En todo caso, el presente artículo no tiene a Derrida como objeto principal de estudio. Como se indica en el resumen, se trata al final del trabajo la crítica derridiana a la distinción de Benjamin entre la violencia instauradora de derecho y la violencia conservadora de derecho. En concreto, se muestra la afinidad de las objeciones que Derrida plantea a Benjamin con la propia crítica de la violencia que cabe encontrar en La estrella de la redención - y que Derrida, a pesar de no mencionarla en Fuerza de ley, conocía con toda seguridad-. Quien escribe se excusa de antemano si ha cometido alguna injusticia con Derrida, cuyo comentario al ensayo benjaminiano no es aquí sometido a un análisis exhaustivo. En este sentido, remito a Gasche (1991) y Weber (1991).

Cf. Benjamin (2007), pp. 183-206.

Cf. Derrida, (2008), pp. 69-140.

Cf. Derrida, (2004), pp. 39-128.

Derrida (2008), p. 75.

Derrida (2008), p. 75.

8 Cf. Derrida (2004), pp. 43 ss. En realidad, lo publicado bajo este título es sólo una parte del seminario, que en su mayoría permanece inédito. Sobre la presencia de Rosenzweig en él, más allá de su relación con Cohen, cf. Hollander (2008). Agradezco esta referencia a Miriam Jerade.

9 Derrida (2008), p. 75.

10 Derrida (2008), p. 75. 
Estado, lo cual significa a su vez que resultará imposible no tener siempre presente a Schmitt. Benjamin, como es de sobra conocido, lo tuvo hasta el final de sus días $\mathrm{y}$, al menos, desde mediados de los años veinte, como tuvo también muy presente, en ese mismo intervalo de tiempo, La estrella de la redención de Rosenzweig, quien sin embargo, hasta donde sabemos, jamás se refirió en sus escritos, ni en sus cartas, a Benjamin -con quien sí conversó en diversas ocasiones, muy a pesar de Scholem-, como tampoco a Schmitt.

\section{Encuentros y desencuentros}

\subsection{Walter Benjamin y Carl Schmitt}

El affaire intelectual entre Benjamin y Schmitt es bastante conocido ${ }^{11}$. En El origen del Trauerspiel alemán, redactado en 1925 como escrito de habilitación y publicado en 1928 una vez su autor había renunciado a la carrera académica, Benjamin se refería hasta en cuatro ocasiones a la Teología política ${ }^{12}$ de Schmitt, a propósito de la importancia de la decisión en la teoría de la soberanía del siglo XVII ${ }^{13}$. Estas citas fueron suprimidas en la edición que apareció en 1955 al cuidado de Theodor W. Adorno. De modo similar, en la edición de la correspondencia de Benjamin, al cuidado en este caso de Gershom Scholem y del propio Adorno, se suprimió la nota que el difunto amigo de ambos había escrito a Schmitt, a finales de 1930, con ocasión del envío de un ejemplar de su frustrado Habilitationsschrift al jurista de Plettenberg. En esta breve misiva, tan célebre ya como inquietante, de la que Jacob Taubes afirmó que se trataba "de una mina que hace sencillamente explotar nuestras representaciones acerca de la historia espiritual del periodo de Weimar"14, Benjamin expresaba a Schmitt -sea esto o no una mera formalidad-su "especial estima", así como la deuda que su libro había contraído con Teología política. Asimismo, se refería al hecho de que había encontrado en obras schmittianas posteriores -sobre todo en el ensayo sobre La dictadura- la confirmación de sus propios métodos de investigación ${ }^{15}$. Estas dos decisiones editoriales, tomadas quizá con la (buena) voluntad de preservar o inmunizar la recepción de Benjamin en Alemania, dado el pasado nacionalsocialista de Schmitt, causaron no obstante un gran revuelo, hasta el extremo de llegarse a acusar a Adorno de falsificación. En la polémica intervinieron Rolf Tiedemann y el propio Scholem, ambos implicados en la edición de las obras de Benjamin, e incluso Hannah Arendt. Del lado de los acusadores destacó ante todo el mencionado Taubes, cuya relación con los albaceas de Benjamin fue siempre muy tensa y que en 1969, de hecho, amenazaba a Scholem con dar difusión a la carta de la discordia, publicada por primera vez en 1970 e incluida en los Gesammelte Schriften de Benjamin en 1974.

También Schmitt, por su parte, citó en una ocasión a Benjamin, a saber, en su Hamlet o Hécuba, publicado en 1956. En este trabajo, a modo de observación

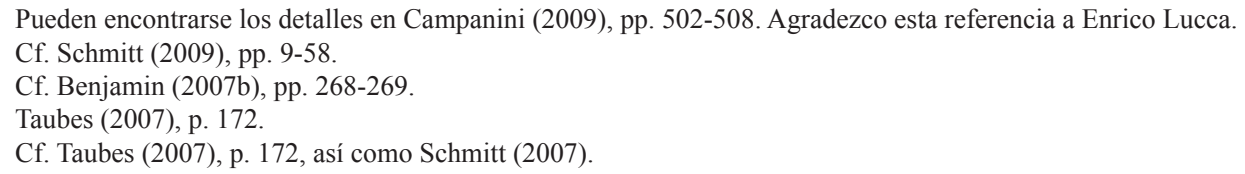


preliminar, Schmitt se refería a El origen del Trauerspiel alemán como un libro que sería útil "al amante de Shakespeare, como al especialista en su obra", y al que él mismo debía "valiosas informaciones y observaciones esenciales"16. Ese mismo año, en carta a Armin Mohler, el apologeta de la Revolución Conservadora alemana, Schmitt se lamentaba de la desaparición de su nombre en la mencionada edición, aparecida un año antes, de la obra de Benjamin, un hecho que tildaba de "escándalo interesante"17. En 1973, publicada ya por tanto la dichosa nota al jurista, éste afirmaba, en privado, que su libro sobre El Leviatán en la teoría del Estado de Thomas Hobbes (1938) había sido una respuesta a Benjamin ${ }^{18}$. En todo caso, Schmitt nunca respondió a la nota de Benjamin, como sin embargo afirma Derrida, quien asimismo alimenta la leyenda -que tiene su origen en Taubes y en el propio Schmitt, quizá como medio para la rehabilitación de la figura de este último- de la existencia de una correspondencia propiamente dicha entre el gran ensayista judío alemán y el gran jurista católico ${ }^{19}$. Sí es más que probable, en cambio, que el autor de Teología política leyese Hacia la crítica de la violencia, toda vez que Schmitt no sólo publicó numerosos textos en el Archiv für Sozialwissenschaften und Sozialpolitik, la revista en la que apareció originalmente el texto de Benjamin, sino que fue lector asiduo de la publicación. En este hecho probable funda Agamben, a su vez, la posibilidad de leer Teología política "como una respuesta precisa al ensayo benjaminiano"20, a saber, como un intento de (re)juridificar la violencia que Benjamin había tratado de no reducir al derecho, mediante la apelación a la existencia de una violencia divina, esto es, destructora del derecho ${ }^{21}$.

Hasta donde se sabe, no hay constancia, en cambio, de que Benjamin hubiese leído a Schmitt antes de y con vistas a la redacción de su trabajo sobre la violencia, pues él mismo reconoce haber leído sólo Teología política, que vio la luz en 1922, así como trabajos posteriores, como se ha mencionado. Cierto es, en todo caso, que La dictadura se publicó de manera original en 1921, pero da la sensación de que la primera obra de Schmitt que fue objeto de lectura por parte de Benjamin fueron, precisamente, sus Cuatro capítulos sobre la doctrina de la soberanía. La tesis principal de este tratado, a saber, la definición del soberano como "quien decide sobre el estado de excepción"22, debió causar un importante impacto en Benjamin, si tenemos en cuenta que aparece mencionada incluso, si bien en esta ocasión de manera velada, en el que fue su testamento espiritual, cuando en la octava de las tesis Sobre el concepto de historia escribe: "La tradición de los oprimidos nos enseña que el 'estado de excepción' en que vivimos es sin duda la regla" ${ }^{23}$. A esta normalización o automatización del estado de excepción, que fue característica, en efecto, del nacionalsocialismo, pero quizá no tanto de la propuesta jurídico-política de Schmitt -que no es solo un pensador de la excepción, sino también de la forma jurídica y del orden concreto, ya desde $1922^{24}$, opone Benjamin "la instauración del estado real

16 Schmitt (1993), p. 5. Schmitt dedica al libro de Benjamin, asimismo, el segundo de los excursos de su escrito. Cf. Schmitt (1993), pp. 51-55.

17 Schmitt-Mohler (1995), p. 218.

18 Cf. Viesel (1988), p. 14 (citado en Agamben (2004), p. 79), así como Schmitt (2004).

19 Cf. Derrida (2008), p. 79, así como Gross, (2005), pp. 12 s., nt. 21.

20 Agamben (2004) pp. $81 \mathrm{~s}$.

21 Cf. Agamben (2004), p. 82, así como Benjamin (2007), pp. 202 s.

22 Schmitt (2009), p. 13.

23 Benjamin (2008), p. 309.

24 Sobre esta cuestión, cf. Navarrete Alonso (2015). 
de excepción”"25, el cual uno tendría la tentación de asimilar a la violencia divina, acaso ya secularizada, del ensayo de $1921^{26}$.

\subsection{Walter Benjamin y Franz Rosenzweig}

La relación entre Benjamin y Rosenzweig resulta más desconocida que la tratada en el anterior apartado. Sin duda, es menos inquietante y escandalosa, salvo quizá para Scholem, como consecuencia de su dificultad para entenderse con Rosenzweig y, añadiría, para entenderlo. Pero incluso él tuvo que reconocer que "Benjamin, como muchos de sus escritos lo demuestran, [fue] un apasionado lector de La estrella de la redención" ${ }^{27}$. El propio Scholem suministró a Benjamin su ejemplar del opus magnum de Rosenzweig, de hecho, y no cabe poner en cuestión la profunda influencia que ejerció en él esta lectura, al menos en lo que tiene que ver con dos cuestiones ${ }^{28}$. En primer lugar, cabe destacar la importancia de la concepción rosenzweiguiana de la tragedia en el libro de Benjamin sobre el Trauerspiel, cuya presencia en esta obra es igual o mayor que la de la teoría de la soberanía de $\mathrm{Schmitt}^{29}$. En segundo lugar, lo cual nos va a interesar en especial aquí, la concepción mesiánica del tiempo histórico que cabe encontrar en la Estrella, y el enfrentamiento con la filosofía de la historia -tanto en su versión metafísica, hegeliana, como en la historicista, rankeana- que ella acarrea, están tan presentes en las tesis benjaminianas Sobre el concepto de historia que, si bien hay constancia de que Benjamin no releyó a Rosenzweig en el tramo final de su vida, sí cabe desde luego pensar que su lectura en años anteriores fue absolutamente determinante para la redacción de este escrito ${ }^{30}$.

\subsection{Franz Rosenzweig y Carl Schmitt}

Mucho más complejo resulta en cambio establecer una conexión directa de Rosenzweig con Schmitt. Aunque resulte sorprendente, dada la relevancia adquirida por Schmitt desde principios de la tercera década del siglo pasado, no cabe encontrar referencia alguna de Rosenzweig al jurista de Plettenberg, tampoco en sus escritos más privados. Incluso Eugen Rosenstock, amigo íntimo del autor de la Estrella, mantuvo contacto con Schmitt, cierto es que después de la muerte de Rosenzweig, durante los años en que ambos coincidieron como profesores de Derecho en la Universidad de Breslau, es decir, inmediatamente antes de la llegada de Hitler al poder y el consiguiente exilio de Rosenstock ${ }^{31}$. Al contrario, por lo demás, cabe encontrar una única referencia de Schmitt a Rosenzweig, en una entrada de 1948 en su Glossarium, sin mayor interés para la cuestión que aquí nos ocupa ${ }^{32}$. En este caso, en definitiva, el problema no tiene tanto que ver con la noción de influencia, ni con los hábitos de lectura tanto de Rosenzweig como de Schmitt, sino que lo decisivo es

25 Benjamin (2008), p. 309.

26 Sobre la relación entre la violencia divina y la revolución en "Hacia una crítica de la violencia", cf. Honneth (2006), pp. 207 ss. Agradezco esta referencia a Miriam Jerade.

27 Scholem (1995), p. 219.

28 El 9 de julio de 1921, en efecto, Benjamin pedía por carta a su amigo Scholem que por favor le enviara la Estrella a Heidelberg, donde por entonces vivía. Cf. Benjamin (1996), p. 164.

29 Cf. Benjamin (2007b), pp. 310, 317, 322 y 326 s.

30 Sobre las afinidades entre ambas obras, cf. Albertini (2005).

31 El propio Schmitt dejó constancia de esta relación. Cf. Schmitt (2010), pp. 86, 108, 111-112 y 255.

32 Cf. Schmitt (1991), p. 153. 
la existencia, como quiso Derrida, de afinidades - y discrepancias- temáticas entre uno y otro, que sí cabe rastrear. La más palmaria tiene que ver con la cuestión de la teología política, a la que Rosenzweig se refiere como "política mesiánica"33, así como con sus respectivas interpretaciones del origen y devenir de la Modernidad. En ambos casos esta genealogía tiene que ver con el concepto de secularización, si bien en dos sentidos diversos y, en realidad, opuestos: como Säkularisierung, si hablamos de Schmitt, y como Verweltlichung, si nos referimos a Rosenzweig. Por último, también en estos dos autores el problema teológico-político se relaciona con lo que cabría caracterizar como una interpretación geo(teo)política de la historia universal a partir de la confrontación de la tierra y el mar ${ }^{34}$.

Respecto de este último punto, merece la pena destacar que, aunque sus análisis son coincidentes en grado sumo, las conclusiones alcanzadas por Schmitt y Rosenzweig acerca del eschaton o final de los tiempos son por completo diversas, tanto y en el mismo sentido que lo son las de Schmitt y Benjamin. El católico, en la línea de la secularización mediante la escatología de la que habla Blumenberg en $L a$ legitimidad de la Edad Moderna ${ }^{35}$, quiso evitar a toda costa la catástrofe en que a su juicio consistiría el eschaton. De ahí la importancia del katechon como presupuesto "filosófico-histórico" de su teoría de la soberanía, al menos desde $1932^{36}$. Por el contrario, los judíos Rosenzweig y Benjamin consideraron la historia que Schmitt quería conservar, justamente, como una catástrofe, de tal manera que su mirada estaba puesta, no en la retención del final de los tiempos, sino en su aceleración, concebida ésta, en todo caso, en términos de anticipación, es decir, nunca de progreso, sino de irrupción y de interrupción, de detención de la historia, siquiera por un instante.

\section{Historia, mesianismo, derecho y justicia}

\subsection{Historia como catástrofe versus temporalidad mesiánica}

La concepción de la historia como catástrofe, por tanto, constituye un primer punto fundamental de coincidencia entre Rosenzweig y Benjamin. Éste lo expresó de manera paradigmática en la archiconocida novena de sus tesis Sobre el concepto de historia:

Hay un cuadro de Paul Klee llamado Angelus Novus. En ese cuadro se representa a un ángel que parece a punto de alejarse de algo a lo que mira fijamente. Los ojos se le ven desorbitados, tiene la boca abierta y además las alas desplegadas. Pues este aspecto deberá tener el ángel de la historia. Él ha vuelto el rostro hacia el pasado. Donde ante nosotros aparece una cadena de datos, él ve una única catástrofe que amontona incansablemente ruina tras ruina y se las va arrojando a los pies. Bien le gustaría detenerse, despertar a los muertos y recomponer lo destrozado. Pero, soplando desde el Paraíso, una tempestad se enreda en sus alas, y es tan fuerte que el ángel no puede cerrarlas. Esta tempestad

\footnotetext{
Cf. Rosenzweig (1997), pp. 389-396.

Cf. Navarrete Alonso (2017).

Cf. Blumenberg (2008), pp. 45-59.

Cf. Grossheutschi (1996).
} 
lo empuja incontenible hacia el futuro, al cual vuelve la espalda mientras el cúmulo de ruina ante él va creciendo hasta el cielo. Lo que llamamos progreso es justamente esta tempestad. ${ }^{37}$

Rosenzweig, por su parte, escribe en términos similares en dos anotaciones redactadas en plena (primera) catástrofe europea, aquella (Primera) Guerra Mundial a la que en buena medida la Estrella quiso ofrecer una respuesta. Las notas en cuestión, de hecho, proceden de los esbozos del nuevo pensamiento que cristalizó en su sistema de filosofía. Ambas fueron escritas el 11 de febrero de 1916. La primera de ellas reza como sigue:

Vista desde una perspectiva naturalista, la eternidad carece de toda relación con el tiempo; no es ni más larga que el tiempo más largo, ni más breve que el tiempo más breve. La Antigüedad (los paganos) dio forma al concepto de lo eterno en este sentido. En consecuencia, no tenían un concepto de historia. En consecuencia, también construyeron este concepto, en primer lugar, en la forma del apocalipsis: la eternidad [como] el final catastrófico del tiempo que irrumpe en mitad del tiempo. Este es todavía el concepto antiguo de la eternidad (incomparable con el tiempo), pero aquí ha entrado en una relación temporal con el tiempo (en tanto que final suyo) - resulta todavía incomparable (aquel mundo), pero en todo caso está ya en una relación. La ampliación racionalista de esta relación consistió en hacer del tiempo el portador de la eternidad: hegelianismo, progresismo, positivismo. La falta antigua de historia se vio de este modo eliminada, pero no así, de manera definitiva, la idea de catástrofe, al menos en el hegelianismo (donde, en cierta medida, cada época se relaciona con la siguiente como este mundo con el mundo por venir). ${ }^{38}$

La segunda anotación es la siguiente:

Toda idea de desarrollo es no apocalíptica. De manera que, ¿no son despreciables entonces, por principio, todas aquellas ideas sobre esta guerra que cuentan con las guerras futuras, es decir, toda política? ¿No debería verse al menos esta guerra como si fuese la última? ¿No hace justo esto la perspectiva chovinista ingenua? [...] ¿O estos "como si" son siempre sólo formas de hablar? El desarrollo no debe ser pensado de manera diferencial, sino catastrófica. Pero, ¿y la multiplicidad de las catástrofes? En efecto, no se puede nunca pensar: infinitud de las catástrofes, sino: "no sabéis el día ni la hora". Lo esencial es, sin

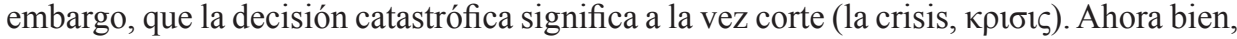
esto significa que la catástrofe individual sólo es absoluta para los individuos y que sólo la última es absoluta para la humanidad. Esto último debe ser pensado en el sentido de que, aquí, el Reino del Bien se consuma, pero también el del Mal - ¡las dos civitates! El mundo, por tanto, deviene siempre tanto mejor como peor. ${ }^{39}$

Los fragmentos referidos, como resulta obvio, ponen de manifiesto un punto en común esencial entre los dos autores judíos alemanes que nos ocupan. Tanto Rosenzweig como Benjamin conciben la historia, no su final, como una catástrofe, lo

\footnotetext{
Benjamin (2008), p. 310.

Rosenzweig (1984), p. 66.

Rosenzweig (1984), pp. $72 \mathrm{~s}$.
} 
cual lleva a ambos, a su vez, a enfrentarse de manera decidida a la idea de desarrollo (Entwicklung) y de progreso (Fortschritt), que como se ha mostrado Rosenzweig asocia de manera inmediata con la filosofía hegeliana de la historia. De especial relevancia es, asimismo, el final de los segundos fragmentos de Rosenzweig citados. En él encontramos, al mismo tiempo, una alusión bastante explícita a Agustín de Hipona y a su doctrina de la dos ciudades, pero también, siquiera de manera implícita, al propio Hegel y, en concreto, a su representación de la historia universal como teodicea, esto es, a su justificación del mal -la catástrofe, o la sucesión de las catástrofes- en la historia en aras de la realización del bien: el progreso en el desenvolvimiento del Espíritu, o de la conciencia de la libertad, según $\mathrm{Hegel}^{40}$. La doble referencia es interesante por varios motivos. En primer lugar, porque Rosenzweig señala aquí con toda claridad que la metafísica hegeliana de la historia significa la disolución dialéctica de la doctrina agustiniana de las dos ciudades, con lo que esto supone desde el punto de vista político, esto es, la eliminación de todo resto entre lo absoluto -la fides, en términos de Agustín de Hipona- y lo relativo -la conservación o salus en el mundo-. Se trata de la supresión de la diferencia que en último término es condición de posibilidad de la resistencia frente a lo que, en términos clásicos, llamaríamos tiranía - tal y como hará Rosenzweig en la Estrella$\mathrm{y}$, en el siglo $\mathrm{xx}$, totalitarismo -o fascismo, como hace Benjamin en sus tesis-. En segundo lugar porque, de hecho, en el fondo el mesianismo de Rosenzweig constituye un regreso a la doctrina agustiniana como medio para denunciar lo excesos de esa política que en la Estrella aparece calificada con el adjetivo "mesiánica"41. Se trata de un gesto, por lo demás, que de modo más o menos velado repetirá en 1935 Erik Peterson, en su caso, contra la teología política de Schmitt ${ }^{42}$. Por último, el fragmento citado con anterioridad debe ser puesto en relación con una carta de Rosenzweig a Hans Ehrenberg, de septiembre de 1910, en la que el autor de la Estrella identifica sin disimulo alguno el sentido de su propio pensamiento:

Vemos a Dios en cada acontecimiento ético, pero no en el todo realizado, en la historia - porque para ello necesitaríamos [...] que la historia fuese divina, que toda acción que ocurriese en esta palangana fuese, sin más, divina, que estuviese justificada. No, toda acción se convierte en pecaminosa en el momento en que entra en la historia [...] y por ello Dios debe redimir al hombre, no a través de la historia, sino, en realidad -no hay otra alternativa-, como "Dios de la religión". Para Hegel la historia era divina, "teodicea" [...]. Para nosotros, la religión es la "única y verdadera teodicea". - La lucha contra la historia en el sentido del siglo XIX es para nosotros, por ello, al mismo tiempo, la lucha por la religión en el sentido del siglo $X x^{43}$

De nuevo, salta a la vista la similitud de las posiciones de Rosenzweig y Benjamin cuando éste, en la primera de las tesis Sobre el concepto de historia, se refiere al enfrentamiento del materialismo histórico con otras concepciones de la historia como uno en el que vencerá "si toma a la teología a su servicio"44. La religión que en Rosenzweig debe luchar contra la historia, y la teología que de acuerdo con Benjamin

40 Cf. Hegel (1980), p. 56.

${ }_{41}$ Sobre la relación de Rosenzweig con la eclesiología agustiniana, cf. Navarrete (2017b), pp. 181 ss.

42 Cf. Peterson (1999).

43 Rosenzweig (1979), pp. 112 s. Las cursivas son mías.

$44 \quad$ Benjamin (2008), p. 305. 
debe enrolar en sus filas el materialismo histórico, en un combate, en ambos casos, contra la ideología del progreso, tienen en verdad uno y el mismo nombre, a saber, el del mesianismo, con toda probabilidad la expresión más elevada de la tradición soteriológica que designamos con el término "judaísmo". Frente al carácter de continuo propio de la concepción de la historia como progreso, al que corresponde un "tiempo homogéneo y vacío" ${ }^{45} \mathrm{o}$, como lo formulará Rosenzweig, un tiempo que no acontece por sí mismo, sino que es la forma a priori -ella misma atemporal-en la que acontecen los acontecimientos, a la temporalidad mesiánica que se describe tanto en Sobre el concepto de historia como en La estrella de la redención le es propio más bien aquel instante que es eterno en la medida en que en él se condensan -y no se suceden- presente, pasado y futuro. El Jetzt-Zeit de Benjamin es un instante dispuesto para la eternidad, "en el cual se han esparcido astillas del mesiánico"46; un nunc stans, como lo describe Rosenzweig en el siguiente pasaje:

Tiene, en efecto, que ser acelerada la eternidad; tiene siempre que poder venir ya "hoy". Sólo gracias a ello es eternidad [...].

¿Qué aspecto habría de tener este hoy? Sobre todo, no podría pasar; porque, aunque de la eternidad no sepamos ninguna otra cosa fuera de ésta, es seguro que ella es lo im-pasable, lo imperecedero. Así que la eternidad transformada en ahora tiene que ser lo primero que le corresponda a este estar determinado por un ahora sin fin. Un hoy imperecedero [...]. El instante le muestra al ojo, como sugiere la expresión alemana para él, Augenblick, ojeada, siempre algo nuevo, tantas veces como el ojo se abra y lance su ojeada. Lo nuevo que nosotros buscamos ha de ser un nunc stans, o sea, no un instante que va de vuelo, sino uno que "está". ${ }^{47}$

La proximidad de Rosenzweig y Benjamin a la hora de concebir la temporalidad mesiánica, y su relación con la temporalidad propia del progreso, es por tanto más que notoria. También ambos concuerdan al señalar los calendarios, que "no cuentan el tiempo como los relojes"48, en los que "la palabra para 'hora' va desligándose de las palabras que designan el tiempo y los períodos en él”49, así como, dentro de ellos, "los días de fiesta" que permite "acelerar el futuro, hacer de la eternidad lo más próximo, el hoy" 53 . Sin duda, los ejemplos aducidos por nuestros autores son distintos: Benjamin está pensando ante todo en la Revolución Francesa, mientras que lo que Rosenzweig tiene en mente es el calendario litúrgico judío y, acaso principalmente, la detención del tiempo que supone la observancia del sabbat. Benjamin, por tanto, está pensando en una versión secularizada de la idea mesiánica, mientras que Rosenzweig sigue concibiendo el mesianismo en términos religiosos. La forma, sin embargo, es la misma, hasta el punto de que, en caso de que se le privase de su contenido,

\footnotetext{
Benjamin (2008), pp. 315 y 318.

Benjamin (2008), p. 318.

Rosenzweig (1997), pp. 344-346.

Benjamin (2008), p. 315.

Rosenzweig (1997), p. 346.

Benjamin (2008), p. 315.

Rosenzweig (1997), p. 347.

Benjamin (2008), p. 316.

Rosenzweig (1997), p. 345.
} 
obtendríamos en ambos casos lo que Derrida nombró con la expresión "mesiánico sin mesianismo" 54 , también, "mesianicidad sin mesianismo" 55 , tratando así, en su empeño deconstructivo, de purificar la estructura mesiánica de todo mesianismo: "de toda traza del Mesías, de judaísmo y de religión en general" ${ }^{56}$. Justo esa estructura es, para Derrida, "irreductible a toda deconstrucción [...], tan indeconstructible como la posibilidad misma de la deconstrucción" ${ }^{57}$ : una "estructura formal de promesa" 58 que, como se ha mostrado, y como nos recuerda el propio Derrida, tiene que ver con el tiempo, con un cierto desajuste temporal o "contratiempo" 59 , pero también, a la vez, con "una idea de la justicia que distinguimos siempre del derecho"60. En ese preciso momento, Derrida remite al lector a su Fuerza de ley, es decir, a Benjamin, pero como trataremos de mostrar también a Rosenzweig.

\subsection{Justicia, derecho y eternidad}

Dos son, en todo caso, las cuestiones que parecen estar aquí en juego, si bien en último término no dejan de ser los dos aspectos de un único problema. Por una parte, se trataría de determinar qué tipo de justicia es esa que corresponde a la temporalidad mesiánica que encontramos tanto en la Estrella como en Sobre el concepto de historia. Lo primero que hay que decir de ella es, en efecto, que se trata de una justicia ubicada al margen de la ley: anómica, por tanto. Así lo sugiere el propio Benjamin, al identificarla, como ya se ha indicado, con un verdadero estado de excepción, por oposición al que es propio del soberano schmittiano: aquel umbral entre lo jurídico y lo político desde el cual se reinstaura o se crea el orden normativo, desde el cual la ley obtiene su fuerza como tal ley. Lo propio de ese verdadero Ausnahmezustand sería, como en el caso de la violencia divina de la que nos habla Benjamin en 1921, la aniquilación del derecho. No parece, sin embargo, que en tanto divina sea una capacidad que esté a nuestra plena disposición, que por tanto esta justa -aunque ilegal, o más bien alegal-, e incluso justísima destrucción de la justicia jurídica pueda realmente ser hecha efectiva, impuesta (enforced) por el ser humano. Más bien, lo que parece ocurrir es que estemos sólo en condiciones de esparcir sus astillas, del mismo modo que el tiempo mesiánico, a decir de Benjamin, acaece sólo como astillado en el "tiempo-ahora". Ninguna revolución es la revolución, sino su anticipación: la prolepsis de una justicia cuya plena realización no está a nuestro alcance, por no ser, a diferencia de nosotros mismos, una justicia de este mundo, sino eterna, es decir, siempre por venir y siempre adviniente. Por otra parte, si su anticipación resulta posible es porque "el mundo es, por un lado, el mundo concreto

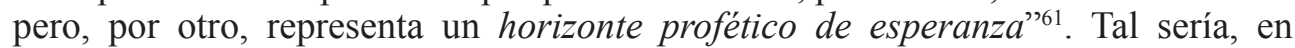
efecto, "el aspecto decisivo del pensamiento rosenzweiguiano de la temporalización, contemplado en perspectiva teológico-política"62.

\footnotetext{
Derrida (2012), p. 73.

Derrida (2005), p. 183.

Bojanic (2018), p. 1.

Derrida (2012), p. 73

Derrida (2012), p. 73.

Cf. Lanceros (2013), así como Bensussan (2001), pp. 124-126.

Derrida (2012), p. 73

Casper (2009), p. 91.

Casper (2009), p. 91.
} 
La segunda de las cuestiones, que en propiedad es la aludida en el título de esta contribución, tendría que ver con la relación entre la justicia del derecho y la eternidad de la que venimos hablando. En este sentido, hemos aclarado ya que esta eternidad no se deja absorber por orden jurídico alguno, sino que permanece de manera esencial fuera de la ley e irreductible a ella. Sin embargo, el derecho aspira a la realización de la justicia, a ser no sólo criterio de la legalidad, sino incluso de la legitimidad. Esta identificación de legalidad y legitimidad es sin duda una característica definitoria de cierto modo de pensar la ciencia jurídica, que si seguimos a Schmitt debemos denominar "normativismo" o "positivismo jurídico"63. Pero el propio Schmitt nos alertó acerca del carácter ideológico del establecimiento de esta identidad. Si legalidad y legitimidad coinciden, ¿desde dónde puede ser puesta en cuestión la justicia del orden legal? ¿O la validez, por ejemplo, de una Constitución? Para Schmitt, nos encontramos aquí ante el núcleo mismo del positivismo jurídico, que reduce el fundamento de la validez del derecho, o de su legitimidad, al significado de la fórmula pacta sunt servanda, respecto del cual el jurista de Plettenberg afirma lo siguiente en su Teoría de la Constitución:

El postulado pacta sunt servanda enuncia que es posible obligarse jurídicamente por medio de pactos. Hoy eso es algo evidente, y no constituye ni una norma, ni el fundamento moral de la validez de normas. Antes bien, o es una completa duplicación e hipóstasis tautológica, o enuncia que el pacto concreto no vale, sino tan solo la "norma" general de que los pactos valen. Adicionar a cada pacto en vigor la "norma" de que los pactos en general son válidos, es una vacua ficción, pues el pacto concreto vale y obliga jurídicamente por virtud del Derecho positivo y no por virtud de la norma pacta sunt servanda. Tales adiciones e hipóstasis ficticias son posibles en número ilimitado; toda norma vale, porque vale la norma general de que hay normas que deben valer, etc. Pero, para la fundamentación de una unidad política de existencia concreta, carecen por completo de significación. ${ }^{64}$

Lo que fundamenta la existencia concreta de una unidad política es, en Schmitt, su Constitución en sentido positivo, a saber, una "decisión de conjunto sobre [el] modo y [la] forma de la unidad política" 65 que ante todo se distingue de la pluralidad de leyes constitucionales recogidas en la lex fundamentalis (concepto relativo de Constitución), así como de esta misma (concepto absoluto), y que a su vez no es la concreción, más o menos perfecta, de ideal alguno (por ejemplo, los de la burguesía en una Constitución concebida como sistema de garantías de la libertad burguesa, distinción de poderes, etc.), sino que, en virtud de su positividad, tiene un carácter, en esencia, concreto, es decir, se refiere a la decisión que una unidad política determinada, que existe de manera efectiva, toma acerca del modo y la forma en que se ordena esta existencia efectiva suya. Se trata, así formulado, de una decisión para la representación de esa unidad política, es decir, para la realización jurídica de un orden concreto prejurídico, ubicado más acá de la ley. Dicha situación previa al derecho, la "normalidad fáctica" de la que habla Schmitt en Teología política, es en último

\footnotetext{
${ }^{63}$ Sobre los diversos modos de pensar la ciencia jurídica, según Schmitt, cf. Schmitt (1996).

${ }_{64}$ Schmitt (1982), p. 87. Sobre la fórmula pacta sunt servanda como fundamento de validez del derecho en el positivismo jurídico, cf. Kelsen (1991).

65 Schmitt (1982), p. 45.
} 
término aquello que confiere legitimidad a la legalidad que lo realiza en el plano jurídico, es decir, que le confiere fuerza de ley. Al decidir sobre la excepción, es en verdad sobre dicha normalidad sobre la que se decide, con vistas a su juridificación. Aquello que distingue la revolución de la contrarrevolución es el hecho de que mientras que esta última trata de conservar, por medio del derecho, la existencia de un determinado orden concreto, aquella apela a una normalidad fáctica distinta a la hasta entonces representada por el derecho y cuya sola existencia legitima o justifica la creación -que por tanto no es ex nihilo- de un nuevo ordenamiento jurídico. En ambos casos, por tanto, se decide sobre el estado de excepción a fin de identificar la normalidad fáctica correspondiente -es decir, la vieja, en un caso, y la nueva, en el otro- y proceder a su juridificación, esto es, a un ajuste entre lo fáctico y lo jurídico a partir de una intervención y, por tanto, un ejercicio de violencia.

\section{Crítica de la crítica benjaminiana de la violencia: Derrida y Rosenzweig}

Como se ve, la crítica benjaminiana de la violencia resulta ser así bastante schmittiana, al menos en lo que a su análisis de la violencia mítica se refiere. En efecto, la distinción de Benjamin entre la "violencia mantenedora de derecho" y la "violencia instauradora de derecho" coincide con la diferencia entre contrarrevolución y revolución que acabamos de presentar a partir del modo en que Schmitt piensa -o dice pensar- la ciencia jurídica. Tal sería, junto al antiparlamentarismo, la afinidad fundamental, al menos en lo que al ensayo sobre la violencia se refiere, entre el gran pensador judío alemán que fue Benjamin y el gran pensador alemán que fue Schmitt, por expresarlo con las palabras de Derrida a las que nos referimos al comienzo de este trabajo ${ }^{66}$. La especial lucidez de Derrida en su comentario al texto de Benjamin se cifra, a mi entender, en el hecho de que ofrece una crítica de la crítica benjaminiana de la violencia, al tiempo que localiza esa misma crítica, o deconstrucción, en el propio escrito de Benjamin. Me refiero a la propuesta derridiana de deconstruir la diferencia entre la violencia que funda el derecho y aquella que la conserva:

Propondría la interpretación según la cual la violencia misma de la fundación o de la posición del derecho (rechtsetzende Gewalt) debe implicar la violencia de la conservación (rechtserhaltende Gewalt) y no puede romper con ella. Forma parte de la estructura de la violencia fundadora el que apele a la repetición de sí y funde lo que debe ser conservado $[\ldots][\mathrm{N}] \mathrm{o}$ hay fundación pura o posición pura del derecho, y en consecuencia pura violencia fundadora, como tampoco hay violencia puramente conservadora. La posición es ya iterabilidad, llamada a la repetición autoconservadora. La conservación a su vez sigue siendo refundadora para poder conservar aquello que pretende fundar. ${ }^{67}$

Como indica Derrida, la distinción entre las dos violencias, que articula la crítica de Benjamin, aparece arruinada en su propio texto, a saber, en las consideraciones que Benjamin presenta respecto de la policía en el Estado moderno, en la cual "está anulada la separación imprescindible de una violencia instauradora de derecho y una

\footnotetext{
66 Respecto de la crítica al parlamentarismo, cf. Benjamin (2007), p. 194, así como Schmitt (2003). El texto de Schmitt data en su versión original de 1923.

67 Derrida (2008), pp. 97 s.
} 
violencia mantenedora del derecho"68. En dicha anulación se cifra, según Benjamin, "lo ignominioso de esa autoridad" 6 . He subrayado el término "imprescindible" porque pone de relieve, con toda evidencia, que en efecto Benjamin no parece estar dispuesto a aceptar esa "mezcla fantasmática" 70 de las dos violencias jurídicas, es decir, la deconstrucción que nos propone Derrida. Considerada desde la lógica schmittiana de la soberanía, la distinción de Benjamin parece de hecho insuficiente. En el extremus necessitatis casus, la conservación del derecho vigente se lleva a cabo mediante su puesta en suspenso, es decir, mediante el retorno al momento de la fundación, que de este modo se convierte eo ipsa en conservadora. Ninguna fundación se hace si no es con vistas al mantenimiento de lo fundado: la violencia conservadora de derecho es (re)fundadora de derecho, y viceversa. O, en otros términos, el Estado moderno es, en esencia, un Estado policial, del mismo modo que toda revolución es de por sí conservadora, y a la inversa: toda contrarrevolución es fundacional. No hay, por tanto, debilitamiento alguno de la violencia instauradora de derecho por parte de la violencia que conserva ese mismo derecho -lo que para Benjamin representa "la ley de su oscilación" "71_, sino, como defiende Derrida, la reafirmación de su fundación mediante la opresión de las violencias que son hostiles a dicha fundación o, lo que es lo mismo, que pretenden ser ellas mismas instauradoras $-\mathrm{y}$ conservadoras- de un nuevo derecho ${ }^{72}$. Lo que debilita la violencia del Estado, que es tanto instauradora como mantenedora de derecho, es, más bien, el transcurrir de la vida de los pueblos hasta el punto en que la contradicción entre orden concreto y orden jurídico resulta insostenible. Es en tales momentos que los estallidos revolucionarios son posibles $y$, en caso de que resulten exitosos, que se alza una nueva época histórica. Para que esto ocurra no basta, entonces, con la suspensión del derecho, sino que es necesaria su renovación. Ésta, empero, envejecerá ipso facto, en el acto mismo de su posición.

La mencionada lucidez de Derrida a la hora de revelar las limitaciones de la crítica benjaminiana de la violencia no es en todo caso original, sino que la deconstrucción de la diferencia entre violencia fundadora y violencia conservadora de derecho se encuentra ya en el apartado de La estrella de la redención dedicado por Rosenzweig a "Los pueblos del mundo"73. Esta sección de la Estrella, obra que por lo demás vio la luz, como el ensayo de Benjamin, en 1921, contiene en efecto, en las secciones rotuladas "El derecho en el Estado" y "La violencia en el Estado"74, una crítica de la violencia muy próxima a la benjaminiana ${ }^{75}$. La exposición por parte de Rosenzweig, sin embargo, adopta una perspectiva filosófico-histórica desde el primer momento ${ }^{76}$.

68 Benjamin (2007), p. 192. El subrayado es mío. Cf. asimismo Derrida (2008), pp. 105 ss.

69 Benjamin (2007), p. 192.

70 Benjamin (2007), p. 192.

71 Benjamin (2007), p. 205.

72 Cf. Benjamin (2007), p. 205.

73 Cf. Rosenzweig (1997), pp. 389-396. Al margen de la cuestión de la originalidad de la crítica que Derrida plantea a Benjamin, lo llamativo es en todo caso que el filósofo francés no se refiera a Rosenzweig en este punto de su comentario al ensayo benjaminiano. No cabe duda de que Derrida conocía la crítica rosenzweiguiana de la violencia a la que vamos a referirnos de inmediato.

74 Rosenzweig (1997), pp. 394 s.

75 Rosenzweig, en todo caso, no pudo conocer el texto de Benjamin antes de escribir La estrella de la redención, cuya redacción se remonta a los últimos meses de la Primera Guerra Mundial. La preparación del manuscrito, en cambio, fue llevada a cabo por Rosenzweig durante 1919, siempre antes de que Benjamin publicase Hacia una crítica de la violencia.

76 También Benjamin, no obstante, adopta este punto de vista, de manera explícita al final de su ensayo, al afirmar que "la crítica de la violencia es ya la filosofía de su historia" (Benjamin (2007), p. 205). Cf. asimismo Honneth 
La crítica rosenzweiguiana de la violencia se plantea desde el problema de la relación del derecho, y del Estado, con la temporalidad de la historia, de este mundo, y con la eternidad suprahistórica del supramundo. No en vano el punto de partida de la exposición de Rosenzweig en este apartado de su obra capital es, como sugiere el propio título, la contraposición del pueblo eterno, cuya temporalidad consiste en una espera que es en sí misma anticipatoria de la eternidad, y, precisamente, los pueblos del mundo, que aspiran a llegar a la meta y están, por consiguiente, en camino. Este camino no es otro que la historia, cuyos portadores son los Estados -el hegelianismo de Rosenzweig en este punto está fuera de toda duda-. Es desde esta perspectiva que Rosenzweig escribe lo siguiente:

El Estado es la forma, siempre cambiante, en que el tiempo va dando paso tras paso hacia la eternidad. En el pueblo de Dios está ya, en mitad del tiempo, lo eterno. En los pueblos del mundo lo que hay es pura temporalidad. Pero el Estado es el intento, necesariamente siempre renovado, de dar a los pueblos eternidad en el tiempo. Ya veremos cómo lo procura. Pero el hecho de que lo procura -y debe procurarlo- lo convierte en imitador y rival del pueblo que en sí mismo es eterno y que ya no tendría derecho alguno a su propia eternidad si pudiera el Estado alcanzar lo que anhela. ${ }^{77}$

La dilucidación del modo en que el Estado procura a los pueblos eternidad en el tiempo representa, justamente, la crítica rosenzweiguiana del derecho, es decir, de la violencia. Porque el derecho es el medio por el cual el Estado retiene, en el sentido del katechon schmittiano, el transcurso de la temporalidad en la que se desenvuelven los pueblos. Pero dicha temporalidad no es otra que la propia de la vida de esos mismos pueblos, de tal modo que la detención del tiempo que procura el Estado por medio del derecho constituye en verdad un atentado contra dicha vida. Sólo en la medida en que la vida lo tolere, el derecho preserva la apariencia de ser sólo derecho:

Pronto el fragor de la vida continúa corriendo [...]. Y derecho y vida, lo permanente y lo mudable, parece que van distanciándose. Entonces es cuando el Estado revela su verdadero rostro. El derecho era nada más que su primera palabra. Esta palabra no puede sostenerse contra el cambio de la vida. Pronuncia ahora su segunda palabra: la palabra de la violencia. ${ }^{78}$

La frase con la que comienza el subapartado siguiente, sobre la violencia en el Estado, es lapidaria: "La violencia restituye a la vida sus derechos contra el Derecho"79. La violencia es, por tanto, el medio del que dispone la vida de un pueblo, configurada de un modo concreto en un momento concreto, para convertirse ella misma en ley, de tal manera que si antes parecía que el derecho era anterior a la violencia, ahora, como antes vimos que ocurría en Benjamin, nos encontramos con lo contrario: "El sentido de toda violencia", escribe Rosenzweig, "es fundar un nuevo derecho. La violencia no es la negación del derecho, como suele pensarse bajo la fascinación de su gesto de subversión, sino, al contrario, su fundamentación" ${ }^{80}$.

\footnotetext{
(2006), pp. 194-197, así como Haverkamp (1991).

Rosenzweig (1997), pp. 393 s.

Rosenzweig (1997), p. 394.

Rosenzweig (1997), p. 394.

Rosenzweig (1997), pp. 394 s.
} 
Sin embargo, a diferencia de lo que ocurría en Benjamin, y era objeto de reproche a éste por parte de Derrida, la renovación y conservación del derecho resultan más bien indistinguibles para Rosenzweig:

En la idea de un derecho nuevo se encuentra una contradicción. El derecho es por su esencia derecho viejo. Y así se manifiesta lo que es la violencia: la renovadora del viejo derecho. En el acto de violencia el derecho se va constantemente convirtiendo en nuevo derecho. Luego el Estado es tanto de derecho como violento: baluarte del antiguo derecho y fuente del nuevo. ${ }^{81}$

La ignominia que Benjamin atribuía a la policía se la hace corresponder Rosenzweig al Estado más de medio siglo antes que Derrida. La empresa del Estado, la introducción de la eternidad en el tiempo, o la retención katechóntica de la historia, consiste para el autor de la Estrella, justamente, en la resolución, a cada instante, de "la contradicción entre lo viejo y lo nuevo gracias a la renovación violenta de lo viejo, que presta a lo nuevo la forma jurídica de lo antiguo" 82 , fundiéndose con ello "al consolidarse osadamente en un "nuevo derecho" "83. Sólo así retiene el movimiento que se escapa: el tiempo; y sólo así es posible hablar de épocas históricas:

Sin Estado no hay, pues, historia del mundo, historia universal. Únicamente el Estado deja caer en el río del tiempo esas imágenes especulares de la verdadera eternidad que constituyen -épocas- los sillares con los que se construye la historia universal. ${ }^{84}$

\section{Conclusión}

A pesar de las inquietantes afinidades que pueden encontrarse entre las concepciones de la soberanía y del Estado moderno de Carl Schmitt, Walter Benjamin y Franz Rosenzweig, existe una diferencia insalvable entre el jurista católico y los dos pensadores judíos alemanes que, en lo fundamental, tiene que ver con la consideración del valor de la historia y de la relación de ésta con el final de los tiempos, es decir, con la relación entre conciencia histórica y conciencia escatológica. Si Schmitt presenta al soberano como aquel que, mediante su decisión sobre la excepción, permite la supervivencia de la historia en la medida en que procura una retención del eschaton, Benjamin y Rosenzweig encuentran en el Estado al portador de una historia que se les muestra como una sucesión injustificada e injustificable de catástrofes. Frente a él y, por tanto, frente a la propia historia y todo intento de justificarla, ambos apelan a una concepción mesiánica de la temporalidad a la que, como supo ver Derrida, corresponde una justicia inasumible por cualquier ordenamiento jurídico, que tanto Benjamin como Rosenzweig, en un ejercicio que podríamos denominar deconstructivo, reducen al ejercicio de violencia en que consiste el poder. Para ambos, el derecho y el Estado representan en definitiva un dispositivo de falsa eternidad

81 Rosenzweig (1997), p. 395.

82 Rosenzweig (1997), p. 395.

83 Rosenzweig (1997), p. 396. Por lo demás, merece la pena señalar aquí que Rosenzweig ya se había ocupado en sus Escritos sobre la guerra, redactados en 1917, del denuedo con el que la política trabaja en la contraposición entre lo viejo y lo nuevo. Cf. Rosenzweig (2015), pp. 37, 39 ss. y 51 ss.

${ }^{84}$ Rosenzweig (1997), p. 396. 
contra el cual únicamente cabe la resistencia posibilitada por la apelación a una justicia que, como la verdadera eternidad, puede tan sólo acaecer en este mundo por anticipado y, nunca, por tanto, de manera definitiva: una justicia cuya consumación significaría al mismo tiempo el final de los tiempos, o la redención.

\section{Referencias bibliográficas}

Agamben, G. (2004): Estado de excepción, Valencia, Pre-textos.

Albertini, F. (2005): "Historia, redención y mesianismo en Franz Rosenzweig y Walter Benjamin. Acerca de una interpretación política de La estrella de la redención”, en Á. E. Garrido Maturano (ed.), El nuevo pensamiento. Seis ensayos introductorios al pensamiento de Rosenzweig, Buenos Aires, Adriana Hidalgo, pp. 127-162.

Benjamin, W. (1996): Gesammelte Briefe. Band II. 1919-1924, Frankfurt a. M., Suhrkamp.

Benjamin, W. (2007): "Hacia la crítica de la violencia”, en Obras. Libro II / Vol. 1, Madrid, Abada, pp. 183-206.

Benjamin, W. (2007b): “El origen del Trauerspiel alemán”, en Obras. Libro I / Vol. 1, Madrid, Abada, pp. 217-459.

Benjamin, W. (2008): "Sobre el concepto de historia", en Obras. Libro I / Vol. 2, Madrid, Abada, pp. 303-318.

Bensussan, G. (2001): Le temps messianique. Temps historique et temps vécu, Paris, Vrin.

Blumenberg, H. (2008): La legitimación de la Edad Moderna, Valencia, Pre-textos.

Bojanic, P. (2018): Violence and Messianism. Jewish Philosophy and the Great Conflicts of the 20th Century, London, Routledge.

Campanini, S. (2009): "Parva Scholemiana IV. Rassegna di Bibliografia", en Materia giudaica. Rivista dell'associazione italiana per lo studio del giudaismo, XIV/1-2, pp. 499-526.

Casper, B. (2009): "Der Andere, der Dritte und die Bürgschaft für die Gerechtigkeit", en Angesichts des Anderen. Emmanuel Levinas - Elemente seines Denkens, Paderborn, Ferdinand Schöningh, pp. 85-96.

Derrida, J. (2012): Espectros de Marx. El estado de la deuda, el trabajo del duelo y la nueva internacional, Madrid, Trotta.

Derrida, J. (2008): "Nombre de pila de Walter Benjamin", en Fuerza de ley. El "fundamento místico de la autoridad”, Madrid, Tecnos, pp. 69-140.

Derrida, J. (2004): “Interpretations at War. Kant, el judío, el alemán“, en Acabados, seguido de Kant, el judio, el alemán, Madrid, Trotta, pp. 39-128.

Derrida, J. (2005): Canallas. Dos ensayos sobre la razón, Madrid, Trotta.

Gasche, R. (1991): “On Critique, Hypercriticism, and Deconstruction: The Case of Benjamin”, en Cardozo Law Review, XIII/4, pp. 1115-1132.

Gross, R. (2005): Carl Schmitt und die Juden. Eine deutsche Rechtslehre, Frankfurt a. M., Suhrkamp.

Grossheutschi, F. (1996): Carl Schmitt und die Lehre vom Katechon, Berlín, Duncker u. Humblot.

Haverkamp, A. (1991): "How to Take it (and Do the Right Thing): Violence and the Mournful Mind in Benjamin's Critique of Violence", en Cardozo Law Review, XIII/4, pp. 11591172.

Hegel, G. W. F. (1980): Lecciones sobre la filosofia de la historia universal, Madrid, Alianza. Hollander, D. (2008): Exemplarity and Choseness. Rosenzweig and Derrida on the Notion of Philosophy, Stanford, Standford University Press. 
Honneth, A. (2006): “'Zur Kritik der Gewalt”, en B. Lindner (ed.), Benjamin Handbuch. Leben-Werk-Wirkung, Stuttgart, J. B. Metzler, pp. 193-210.

Kelsen, H. (1991): "Por qué obedecer al Derecho?”, en ¿Qué es justicia?, Barcelona, Ariel, pp. 183-193.

Lanceros, P. (2013): “Contratiempo. De un borrón de tinta en el papel secante”, en J. Barja - C. Rendueles (eds.), Mundo escrito. 13 derivas desde Walter Benjamin, Madrid, CBA, pp. 165-200.

Navarrete Alonso, R. (2015): “Carl Schmitt y el pensamiento del orden concreto: una crítica de la interpretación decisionista de la teología política schmittiana”, en Isegoría. Revista de Filosofía Moral y Politica, 52, pp. 349-364.

Navarrete Alonso, R. (2017): “Geo(teo)política de la historia universal. Franz Rosenzweig y Carl Schmitt”, en Éndoxa. Series filosóficas, 40, pp. 183-202.

Navarrete Alonso, R. (2017b): "Rosenzweig adversus Marción: sinagoga, iglesia, mundo", en F. Rosenzweig y E. Rosenstock, Cartas sobre judaísmo y cristianismo, Salamanca, Sígueme, pp. 155-185.

Peterson, E. (1999): El monoteísmo como problema político, Madrid, Trotta.

Rosenzweig, F. (1979): Der Mensch und sein Werk. Gesammelte Schriften I. Briefe und Tagebücher und Tagebücher 1 (1900-1918), Haag, Martinus Nijhoff.

Rosenzweig, F. (1984): "Paralipomena", en Der Mensch und sein Werk. Gesammelte Schriften III. Zweistromland. Kleinere Schriften zu Glauben und Denken, Haag, Martinus Nijhoff, pp. 61-124.

Rosenzweig, F. (1997): La estrella de la redención, Salamanca, Sígueme.

Rosenzweig, F. (2015): Escritos sobre la guerra, Salamanca, Sígueme.

Schmitt, C. (1982): Teoría de la Constitución, Madrid, Alianza.

Schmitt, C. (1991): Glossarium. Aufzeichnungen der Jahre 1947-1951, Berlin, Duncker u. Humblot.

Schmitt, C. (1993): Hamlet o Hécuba. La irrupción del tiempo en el drama, Murcia, Pretextos.

Schmitt, C. (1996): Sobre los tres modos de pensar la ciencia jurídica, Madrid, Tecnos.

Schmitt, C. (2004): El Leviatán en la Teoría del Estado de Tomás Hobbes, Granada, Comares.

Schmitt, C. (2007): La dictadura desde los comienzos del pensamiento moderno de la soberanía hasta la lucha de clases proletaria, Madrid, Alianza.

Schmitt, C. (2008): Los fundamentos histórico-espirituales del parlamentarismo en su situación actual, Madrid, Tecnos.

Schmitt, C. (2009): "Teología política. Cuatro capítulos sobre la doctrina de la soberanía”, en Teología política, Madrid, Trotta, pp. 9-58.

Schmitt, C. (2010), Tagebücher. 1930-1934, Berlin, Akademie Verlag.

Schmitt, C. - Mohler, A. (1995): Briefwechsel mit einem seiner Schüler, Berlin, Akademie Verlag.

Scholem, G. (1995): “Walter Benjamin”, en Judaica 2, Frankfurt a. M., Suhrkamp, pp. 193227.

Taubes, J. (2007): La teología política de Pablo, Madrid, Trotta.

Viesel, H. (1988): Jawohl, Herr Schmitt. Zehn Briefe aus Plettenberg, Berlin, Support.

Weber, S. (1991): "Deconstruction before the Name: Some Preliminary Remarks on Deconstruction and Violence", en Cardozo Law Review, XIII/4, pp. 1181-1190. 